\section{Protein Expression Both in Mammalian Cell Lines and in Yeast Pichia pastoris Using a Single Expression Plasmid}

BioTechniques 24:266-271 (February 1998)

\begin{abstract}
We have designed and constructed a novel expression vector capable of producing recombinant proteins in both mammalian cell lines and the yeast strain Pichia pastoris. In this vector, a yeast promoter is placed inside an intron of the mammalian transcription unit. A yeast transcription termination sequence is placed immediately downstream of the mammalian polyadenylation site. In mammalian cells, transcription is driven by a mammalian promoter. The yeast promoter within the intron is removed by RNA processing. However, protein expression in yeast cells can be achieved utilizing the yeast promoter immediately upstream of the $3^{\prime}$ splice site and the target genes. Our data indicate that this vector can express $\beta$-galactosidase efficiently in both mammalian cell lines and the yeast strain P. pastoris.
\end{abstract}

\section{INTRODUCTION}

Mammalian cell lines and yeast are two commonly used host systems for the expression of recombinant gene products (9). Yeast systems are easy to handle and usually express high levels of proteins, while mammalian expression systems may produce more native biological materials. However, both systems have their limitations. For example, mammalian proteins produced in yeast will have different glycosylation patterns from their native form. Conversely, establishing a mammalian cell line for protein production is time-consuming, and the yield of product is relatively low in comparison with yeast. Choosing a proper system for protein expression has been an empirical matter.

The Pichia pastoris yeast strain is one of those most commonly used in protein expression, due to its high pro- ductivity and convenient media selection. To achieve protein expression in mammalian and yeast systems such as $P$. pastoris, two separate expression plasmids must be constructed. Therefore, it would be desirable to design a shuttle expression vector that would allow exploration of protein expression in both systems quickly. We have developed such a plasmid vector and succeeded in using this vector to express $\beta$-galactosidase ( $\beta$-gal) in several mammalian cell lines and in the yeast strain, $P$. pastoris.

\section{MATERIALS AND METHODS}

\section{Plasmid Construction}

The yeast $P$. pastoris expression vector pHIL-D2 was obtained from Invitrogen (Catalog No. K1710-01; Carlsbad, CA, USA). A fragment containing the mammalian promoter $\mathrm{SR} \alpha$ (from the DNAX Research Institute of Molecular and Cellular Biology, Palo Alto, CA, USA; and Reference 10) and the human $\beta$-globin $5^{\prime}$ splice site was cloned into the NdeI site of pHIL-D2 blunt-ended by Klenow enzyme. Another fragment containing an immunoglobulin $3^{\prime}$ splice site, a polylinker and a simian virus 40 (SV40) late polyadenylation signal was then inserted into the EcoRI site of the previous construct blunt-ended with Klenow enzyme. The resulting vector was named

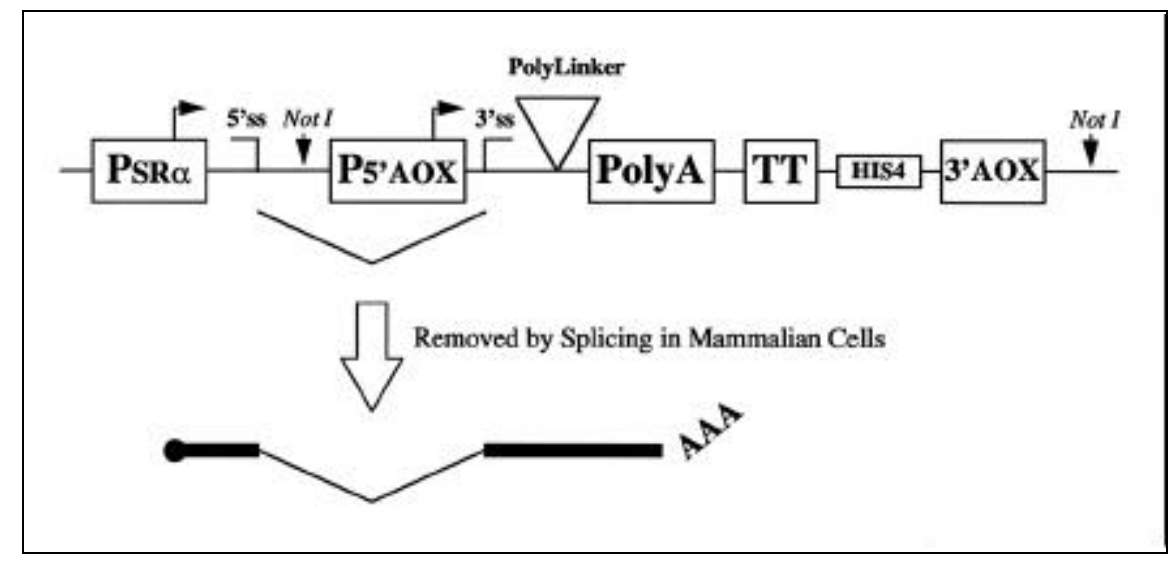

Figure 1. A schematic diagram of the expression units of pPM-2. The construction of the vector was described in Materials and Methods. The yeast promoter P5'AOX was placed within the intron of the mammalian transcription unit. The yeast TT was placed downstream of the mammalian polyadenylation site [Poly(A)]. In mammalian cells, the entire intron including the P5'AOX should be removed from the mature mRNA. Before transformation into yeast $P$. pastoris GS115, the vector was digested with NotI as indicated (not drawn to scale).
pPM-2. To insert a target gene, an SmaI-DraI fragment from pSV $\beta$ (Cata$\log$ No. 6178-1; CLONTECH Laboratories, Palo Alto, CA, USA) containing a $\beta$-gal cDNA was cloned into the SmaI site of pPM-2 (pPM-2- $\beta$ gal). The above constructs were mapped with multiple restriction digestions and confirmed by DNA sequencing analysis. To generate riboprobes used in the subsequent RNase protection assay, plasmid R-6 was constructed by inserting a $P v u \mathrm{I}-H i n d I I I$ fragment of pPM-2- $\beta$ gal into the $E c o \mathrm{RV}$ site of a pBluescript ${ }^{\circledR}$ SK II vector (Stratagene, La Jolla, CA, USA). The insert spans across the $3^{\prime}$ splice site of pPM-2- $\beta$ gal.

\section{Yeast and Mammalian Cell Culture}

The yeast $P$. pastoris strain GS115 (K1710-01) was obtained from Invitrogen. Its propagation, competent cell preparation and selection procedures followed the manufacturer's recommendations. Before electroporation into competent GS115 cells, the pPM-2$\beta$ gal was digested with NotI to release the yeast expression cassette. Mammalian cells were maintained following standard tissue-culture techniques. Culture media for individual cell lines followed ATCC (Rockville, MD, USA) recommendations. Supercoiled pPM-2$\beta$ gal $(10 \mu \mathrm{g})$ was transfected, using the calcium phosphate precipitation protocol (8), into cells on $10-\mathrm{cm}$ tissue culture dishes. 


\section{Assays for $\beta$-gal Expression}

For the yeast $P$. pastoris, selected clones were scaled up for production. Each was sampled at different time points, as indicated in Figure 2. Cell lysates were prepared according to the manufacturer's protocols. For mammalian cells, total cell lysates were prepared $48 \mathrm{~h}$ after transfection. Amounts of $\beta$-gal produced by yeast and different mammalian cell lines were quantitated by an enzyme-linked immunosorbent assay (ELISA) kit (Catalog No. 1 539 426; Boehringer Mannheim, Indianapolis, IN, USA). The enzymatic activity was measured with the $\beta$ Galactosidase Enzyme Assay System (Promega, Madison, WI, USA). Protein concentrations were measured by BCA $^{\mathrm{TM}}$ Protein Assay (Pierce Chemical, Rockford, IL, USA).

\section{RNA Isolation and RNase Protection Assay}

Poly(A+) RNA samples were isolated from CHO-K1 cells $48 \mathrm{~h}$ after transfection with pPM-2- $\beta$ gal $\left(\right.$ Oligotex ${ }^{\mathrm{TM}}$ mRNA Isolation Kit; Qiagen, Chatsworth, CA, USA). R-6 was linearized by $X b a \mathrm{I}$ digestion and in vitro-transcribed with T7 DNA polymerase. Approximately $1 \mu \mathrm{g}$ of poly(A+) RNA sample was used for the RNase protec-

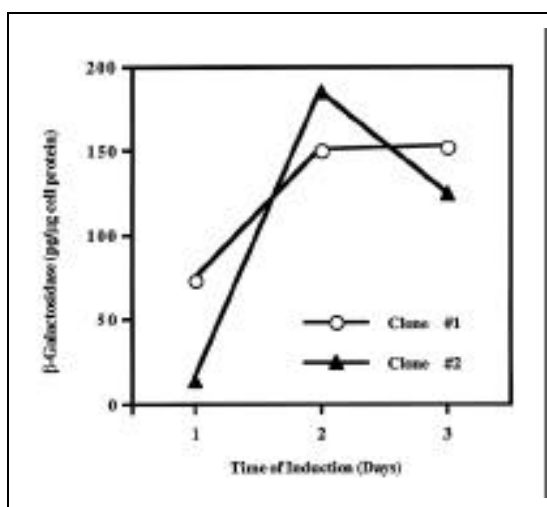

Figure 2. $\beta$-gal protein expression in yeast $\boldsymbol{P}$. pastoris. The GS115 strain of yeast $P$. pastoris was transformed with pPM-2- $\beta g a l$. After His selection, two clones expressing $\beta$-gal were identified and maintained for 3 days under continuous methanol induction. Yeast samples were collected at various time points, as indicated. Cell lysates were prepared to assay for $\beta$-gal production by ELISA. The amounts of $\beta$-gal were normalized against total cellular protein. These yeast lysates were found to be enzymatically active in a separate experiment (data not shown). tion assay, supplemented with $20 \mu \mathrm{g}$ of total RNA from untransfected CHO-K1 cells (Catalog No. 1410; Ambion, Austin, TX, USA). Digested samples were resolved on a $6 \%$ denaturing polyacrylamide gel.

\section{RESULTS}

\section{Vector Design}

Splicing of mRNA requires a pair of splice sites on the nascent mRNA precursor. These sites are cleaved and rejoined at adjacent exons to remove the non-coding intron from the mature mRNA $(5,7)$. This process is required for the export, stability and eventually the accumulation of most protein-encoding mRNA species in the cytoplasm of mammalian cells (1). In our vector design, the yeast promoter $\mathrm{P}^{\prime}$ AOX was placed between the $5^{\prime}$ and $3^{\prime}$ splice sites so that it would be removed as part of the intron during splicing when transfected into mammalian cells (Figure 1). The removal of yeast promoter allows efficient expression from the transcription unit driven by the $\mathrm{SR} \alpha$ promoter. Moreover, in P. pastoris yeast, both the mammalian promoter and the $5^{\prime}$ splice site are removed by NotI digestion before electroporation. The same cDNA fragment downstream of the 3' splice site can be transcribed from the $5^{\prime}$ AOX promoter. The $3^{\prime}$-splice-site region be- tween the polylinker and the P5'AOX was engineered to remove any potential translation initiation codons (ATG). In addition, the transcription termination (TT) element of the yeast shares no apparent homology with that of the mammalian Poly(A) signal (6). Thus, there should be no interference between the two promoter elements and the two termination signals.

\section{Expression of $\beta$-gal Protein in Yeast P. pastoris}

A total of five clones of $P$. pastoris transformed with pPM-2- $\beta$ gal were expanded for assay of $\beta$-gal protein expression after His selection. Two were positive by ELISA. Both cultures were further sampled at various times in the following experiment. The amounts of $\beta$-gal produced were measured by ELISA (Figure 2). The $\beta$-gal enzymatic activity of these samples was confirmed in a separate experiment (data not shown). Our data indicate that both clones have a similar level of $\beta$-gal expression and are stable for at least three days.

\section{Expression of $\beta$-gal in Mammalian Cell Lines}

Six mammalian cell lines were tested for $\beta$-gal expression from pPM-2$\beta$ gal. All except for HeLa S3 expressed $\beta$-gal. The $\beta$-gal production was

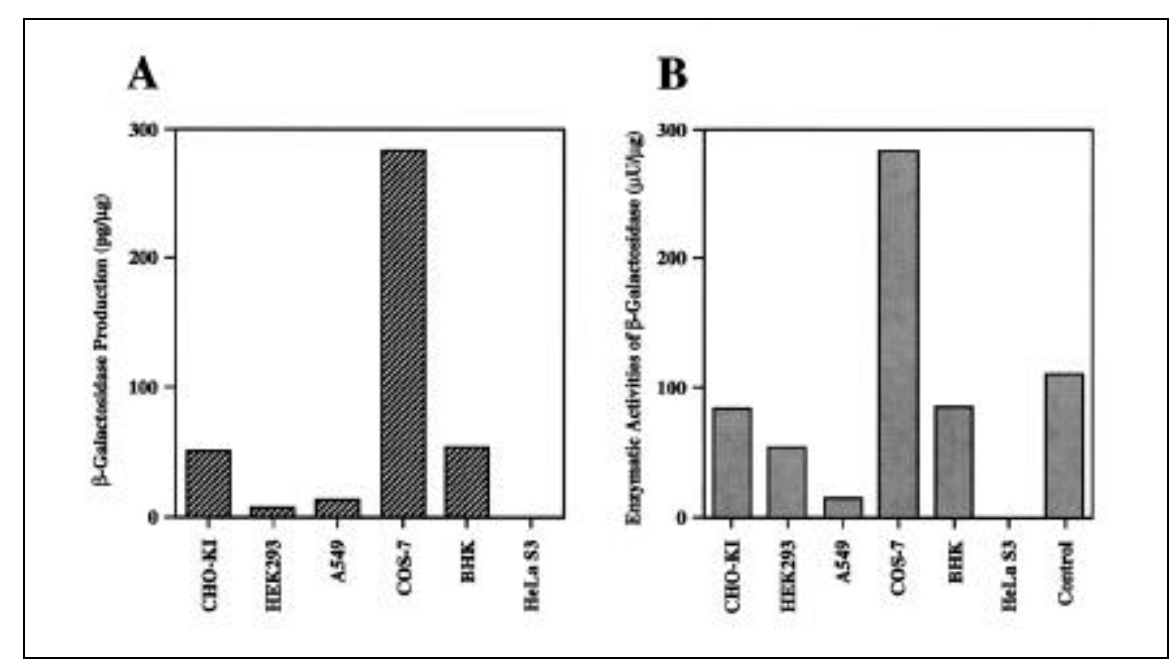

Figure 3. $\beta$-gal expression in mammalian cell lines. The indicated mammalian cell lines were transiently transfected with pPM-2- $\beta$ gal. Cell lysates were prepared $48 \mathrm{~h}$ after transfection. The amounts of $\beta$-gal protein expressed were measured by ELISA (A) and by enzymatic activity (B). The numbers were normalized against total cellular protein. Positive control lane in Panel B: CHO-K1 cells transfected with pCMV $\beta$ (Catalog No. 6177-1; CLONTECH). 


\section{Short Technical Reports}

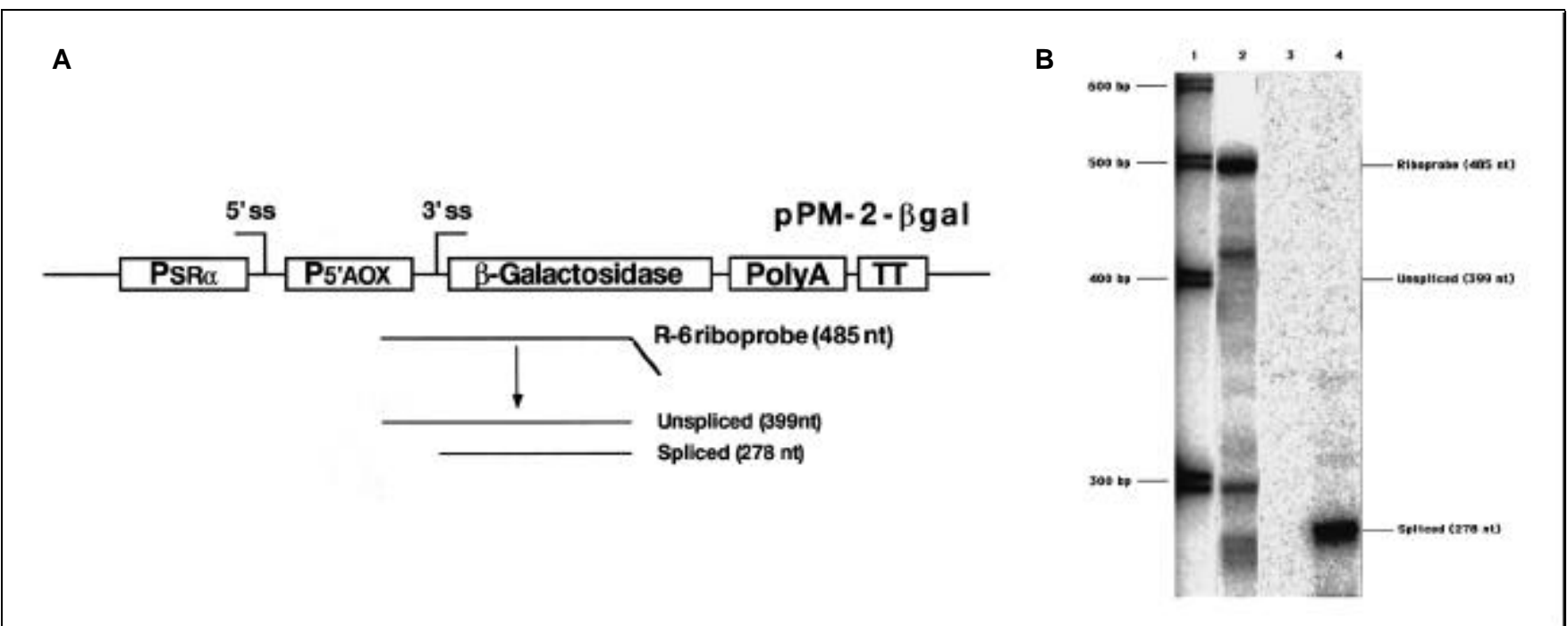

Figure 4. RNase protection assay of $\boldsymbol{\beta}$-gal mRNA from CHO-K1 cells transfected with pPM-2- $\beta$ gal. (A) The riboprobe generated from clone R-6 spans across the 3' splice site of pPM-2- $\beta$ gal. Predicted sizes of riboprobe, spliced and unspliced fragments, are indicated (not drawn to scale). (B) Results from the RNase protection assay with riboprobe R-6. Lane 1: end-labeled 100-bp DNA ladder; lane 2: undigested riboprobes; lane 3: CHO-K1 cells transfected with pBluescript SK II; and lane 4: CHO-K1 cells transfected with pPM-2-ßgal.

measured simultaneously by both ELISA and its enzymatic activities (Figure 3). Among the cell lines tested, the highest level of $\beta$-gal expression was seen with COS-7 cells, presumably due to the presence of an SV40 promoter in the plasmids and a large $T$ antigen expressed in COS-7 cells.

\section{Removal of the $5^{\prime}$ AOx Promoter in CHO-K1 Cells}

To verify the efficiency and accuracy of removal of the $5^{\prime}$ AOX promoter by RNA splicing, an RNase protection assay with a riboprobe (R-6) spanning the 3 -splice-site region was performed (Figure 4A). In particular, a ${ }^{32} \mathrm{P}$-labeled riboprobe was annealed to mRNA sample isolated from CHI-K1 cells trans-

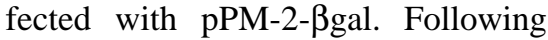
RNase digestion, the sample was resolved on a denaturing polyacrylamide gel. Results showed no detectable level of unspliced mRNA from the poly(A+) RNA sample (Figure 4B). This indicates that the intron is efficiently removed from the mRNA molecules. The excision occurs precisely at the $3^{\prime}$ splice site indicated (Figure 4).

\section{DISCUSSION}

The number of genes identified has grown rapidly in recent years. Many of the gene products need to be expressed as either wild-type or mutated forms in several biological systems, to study their structure and function. To meet this challenge, we have constructed a dual mammalian/yeast expression vector that is capable of expressing recombinant proteins in both host systems. The organization of this vector allows a yeast (P. pastoris) transcription unit to function independently from an overlapping mammalian expression cassette. In mammalian cells, the transcript derived from the $S R \alpha$ promoter is properly processed and accumulated in the cytoplasm for translation. Our data have shown that splicing efficiently removed yeast promoter elements from poly(A+) mRNA (Figure 4 and Reference 3). In yeast $P$. pastoris, gene expression is controlled by the yeast $5^{\prime}$ AOX promoter. The transforming plasmid is linearized at NotI sites, thereby disrupting the mammalian expression cassette. The mammalian polyadenylation site will be ignored, since the SV40 poly(A) signal shares no homology with the TT sequence of the yeast.

It is foreseeable that more shuttle expression vectors can be built based on a similar design. For example, a combination of the T7 promoter and a mammalian promoter can achieve protein expression in both E. coli and mammalian cell lines. Furthermore, a selection marker can also be incorporated into the vector to allow the establishment of permanent clones expressing desired targets in mammalian cells $(2,4)$.

\section{REFERENCES}

1.Buchman, A.R. and P. Berg. 1988. Comparison of intron-dependent and intron-independent gene expression. Mol. Cell Biol. 8:43954405.

2.Kaufman, R.J., M.V. Davies, L.C. Wasley and D. Michnick. 1991. Improved vectors for stable expression of foreign genes in mammalian cells by use of the untranslated leader sequence from EMC virus. Nucleic Acids Res. 19:4485-4490.

3.Liu, Z., L.M. Cashion, L. Zhu and Y. Luo. 1997. A mammalian gene expression vector with blue-white selection for efficient subcloning in E. coli. Anal. Biochem. 246:264267.

4.Lucas, B.K., L.M. Giere, R.A. DeMarco, A. Shen, V. Chisholm and C.W. Crowley. 1996. High-level production of recombinant proteins in CHO cells using a dicistronic DHFR intron expression vector. Nucleic Acids Res. 24:1774-1779.

5.McKeown, M. 1992. Alternative mRNA splicing. Annu. Rev. Cell Biol. 8:135-155.

6.Proudfoot, N.J. 1991. Poly(A) signals. Cell 64:671-674.

7.Rio, D.C. 1992. RNA processing. Curr. Opin. Cell Biol. 4:444-452.

8.Sambrook, J., E.F. Fritsch and T. Maniatis. 1989. Introduction of recombinant vectors into mammalian cells, p. 16.30-16.40. Molecular Cloning: A Laboratory Manual, 2nd ed. CSH Laboratory Press, Cold Spring Harbor, NY.

9.Shatzman, A.R. 1995. Expression systems. Curr. Opin. Biotechnol. 6:491-493.

10.Takebe, Y., M. Seiki, J. Fujisawa, P. Hoy, K. 
Yokota, K. Arai, M. Yoshida and N. Arai 1988. SR alpha promoter: an efficient and versatile mammalian cDNA expression system composed of the simian virus 40 early promoter and the R-U5 segment of human T-cell leukemia virus type 1 long terminal repeat. Mol. Cell Biol. 8:466-472.

The authors acknowledge the DNAX Research Institute of Molecular and Cellular Biology for providing the plasmid $p c D L$ SR 2 296. Address correspondence to Dr. Zhong Liu, Berlex Biosciences, 15049 San Pablo Avenue, P.O. Box 4099, Richmond, CA 94804-0099, USA. Internet: zhong_liu @berlex.com

Received 25 April 1997; accepted 8 October 1997.

Zhong Liu, Linda M. Cashion and Haifeng Pu

Berlex Biosciences

Richmond, CA, USA

\section{RNA Quantitative Analysis from Fixed and Paraffin-Embedded Tissues: Membrane Hybridization and Capil- lary Electrophoresis}

BioTechniques 24:271-276 (February 1998)

\begin{abstract}
Fixed and paraffin-embedded tissues from pathology department archives are available for RNA expression analysis. We describe a general method for quantitation of specific RNA sequence extracted from single 6-8- $\mu m$ human histological tissue sections cut from paraffin blocks. For each specific $m R N A$, the range of linear relationship between the log of the initial total RNA concentration and the log of the specific product after reverse transcription (RT)PCR must be established. We usually perform RT with avian myeloblastosis virus $(A M V)-R T$, using specific antisense primers and a variable number of cycles of PCR
\end{abstract}

amplification. The number of cycles must be adjusted within the range in which a linear relationship exists between the log of the amount of amplification product and the number of cycles. The quantity of specific product is standardized relative to $\beta$-actin mRNA to normalize for the degree of RNA degradation, which can be quite different among samples. The amplification products were quantified by dot blot and ${ }^{32} P$-labeled hybridization probe or by capillary electrophoresis with a laser-induced fluorescence detector. The intratest variation range was for the dot blot mean $\pm 10 \%$ standard deviation (SD) and for the capillary electrophoresis mean $\pm 3 \% S D$.

\section{INTRODUCTION}

Recent studies have shown the possibility of analyzing RNA extracted from fixed and paraffin-embedded tissues $(5,14,16)$. A specific RNA analysis in embedded tissues facilitates the utilization of human pathological tissues. In fact, all bi-optical or surgical tissues are routinely fixed and paraffinembedded and conserved for many years.

Some of the methods proposed (14) for the RNA extraction utilize single 6-8- $\mu \mathrm{m}$ histological sections from paraffin blocks. The RNA is first transcribed into cDNA and subsequently amplified with the polymerase chain reaction (PCR) technology. Methods to quantify RNA extracted from fresh tissues after PCR amplification have already been proposed $(2,7,10)$. However, the quantitation of RNA from paraffin-embedded tissues presents specific problems that cannot be resolved with a quantitative competitive analysis because of the variable level of RNA degradation in the samples. We describe a relative quantitative analysis of specific RNA sequences from fixed and paraffin-embedded human tissues, comparing the membrane hybridization $(\mathrm{MH})$ and capillary electrophoresis (CE) methods.

\section{MATERIALS AND METHODS}

Human tissues from surgical or autopsy origin were fixed with $10 \%$ buffered formalin and then paraffin- 